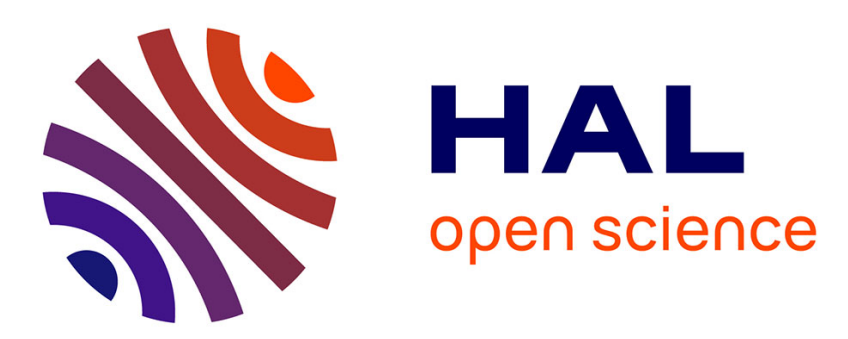

\title{
A Note on the Complexity of Classical and Intuitionistic Proofs
}

\author{
Matthias Baaz, Alexander Leitsch, Giselle Reis
}

\section{To cite this version:}

Matthias Baaz, Alexander Leitsch, Giselle Reis. A Note on the Complexity of Classical and Intuitionistic Proofs. LICS 2015 - 30th Annual ACM/IEEE Symposium on Logic in Computer Science, Jul 2015, Kyoto, Japan. pp.657 - 666, 10.1109/LICS.2015.66 hal-01208346

\section{HAL Id: hal-01208346 \\ https://hal.inria.fr/hal-01208346}

Submitted on 2 Oct 2015

HAL is a multi-disciplinary open access archive for the deposit and dissemination of scientific research documents, whether they are published or not. The documents may come from teaching and research institutions in France or abroad, or from public or private research centers.
L'archive ouverte pluridisciplinaire HAL, est destinée au dépôt et à la diffusion de documents scientifiques de niveau recherche, publiés ou non, émanant des établissements d'enseignement et de recherche français ou étrangers, des laboratoires publics ou privés. 


\section{A note on the complexity of classical and intuitionistic proofs}

\author{
Matthias Baaz \\ Institute of Discrete Mathematics and Geometry \\ Vienna University of Technology \\ baazelogic.at
}

\author{
Alexander Leitsch \\ Institute of Computer Languages \\ Vienna University of Technology \\ leitschelogic.at
}

\author{
Giselle Reis \\ Inria Saclay, France \\ giselle.reiseinria.fr
}

\begin{abstract}
We show an effective cut-free variant of Glivenko's theorem extended to formulas with weak quantifiers (those without eigenvariable conditions): "There is an elementary function $f$ such that if $\varphi$ is a cut-free LK proof of $\vdash A$ with symbol complexity $\leq c$, then there exists a cut-free $\mathbf{L J}$ proof of $\vdash \neg \neg A$ with symbol complexity $\leq f(c)$ ". This follows from the more general result: "There is an elementary function $f$ such that if $\varphi$ is a cut-free $\mathbf{L K}$ proof of $A \vdash$ with symbol complexity $\leq c$, then there exists a cut-free $\mathbf{L J}$ proof of $A \vdash$ with symbol complexity $\leq f(c)$ ". The result is proved using a suitable variant of cut-elimination by resolution (CERES) and subsumption.
\end{abstract}

\section{INTRODUCTION}

In mathematics there are theorems whose proofs can be drastically shortened by working in more powerful axiom systems. The first result of this kind has been the Gödel speed-up theorem [1]-[3], an effective variant of the second incompleteness theorem (some natural examples of this phenomenon can be found in [4]). The most general speedup result in the literature is the theorem of Ehrenfeucht and Mycielski [5]:

If the theory $T+\neg \alpha$ is undecidable, then there is no recursive function $f$ such that $\|A\|_{T} \leq$ $f\left(\|A\|_{T+\alpha}\right)$, for every sentence A provable in $T$.

Where $\|S\|$ denotes the symbol complexity of $S$ and $\|A\|_{T}$ is the proof complexity of $A$ in $T$, i.e. the minimal size of a proof of $A$ in $T$. Note that, for $T+\neg \alpha$ being undecidable, it is necessary that $\alpha$ is not a theorem of $T$, otherwise $T+\neg \alpha$ would be inconsistent and therefore decidable.

The Ehrenfeucht-Mycielski theorem is formulated prima facie for classical logic systems, but holds for intuitionistic logic systems as well. Consider that $T$ is intuitionistic logic (IL) and let $\alpha$ be a sentence $A$ such that $I L+\neg A$ is undecidable. Take the sequents $S_{B}=A \vdash \neg A \rightarrow B$ for all first-order sentences $B$. Obviously, there are recursively bound proofs of the $S_{B}$ in $I L$ and thus recursively bound proofs of the formulas $\neg A \rightarrow B$ in $I L+A$. Assume that there is a function $f$ such that $\|C\|_{I L} \leq f\left(\|C\|_{I L+A}\right)$ for every sentence $C$. Then if we take $C_{B}=\neg A \rightarrow B$, it must be the case that the formulas $C_{B}$ have recursively bound proofs in $I L$. But note that this would mean that the formulas $B$ have recursively bound proofs in $I L+\neg A$, contradicting its undecidability.

The Ehrenfeucht-Mycielski theorem however does not apply to the relation of intuitionistic and classical first-order logic, as intuitionistic logic extended by the negation of any instance of the tertium non datur is in fact inconsistent and therefore decidable. Nevertheless, there are non-recursively bounded speed-ups of classical over intuitionistic logic. Take for example the sentences $F_{B}: B \vee \neg B$, where $B$ ranges over all intuitionistically valid first-order sentences. Clearly all $F_{B}$ have bounded classical proofs (linear in $\|B\|$, the size of $B$ ). On the other hand there exists an infinite sequence $B_{n}$ of intuitionistically provable formulas for which there exists no recursive function $f$ with $\left\|B_{n}\right\|_{\mathbf{L J}} \leq f\left(\left\|B_{n}\right\|\right)$ for all $n$ (follows from the undecidability of first-order intuitionistic logic). This gives us the following speed-up result: there exists no recursive function $f$ such that $\|A\|_{\mathbf{L K}} \leq f\left(\|A\|_{\mathbf{L J}}\right)$ for all formulas $A$ provable in intuitionistic first-order logic. Another example of a nonrecursive speed-up by classical over intuitionistic logic is the monadic fragment [6]. In general it is therefore interesting to investigate the general conditions under which speed-ups can be achieved.

On the relation of classical and intuitionistic proofs, the best known result is Glivenko's theorem, also called double negation translation:

An arbitrary propositional formula $A$ is classically provable if and only if $\neg \neg A$ is intuitionistically provable.

In this paper we consider the extension of Glivenko's double negation translation to formulas $A$ with weak quantifiers only. Note that Glivenko's theorem does not hold if strong quantifiers are admitted, e.g, $\forall x . \neg \neg A(x) \rightarrow \forall x . A(x)$ is classically provable but $\neg \neg(\forall x . \neg \neg A(x) \rightarrow \forall x . A(x))$ is not intuitionistically provable in general. There is of course a polynomial double negation translation with respect to the fragment with weak quantifiers of cut-free $\mathbf{L K}$ proofs into LJ proofs, but this translation introduces cuts (see Section IV-A). Therefore, it does not provide an elementary bound with respect to the relation of cut-free proofs.

The main result of this paper is the following: there is an elementary function $f$ such that if $\varphi$ is a cut-free $\mathbf{L K}$ proof of $A \vdash$ with symbol complexity $\leq c$, then there exists a cutfree $\mathbf{L J}$ proof of $A \vdash$ with symbol complexity $\leq f(c)$. An 
elementary bound for the double negation translation follows immediately. As a corollary we obtain: if subclasses of $\mathbf{L K}$ proofs of sequents $A \vdash$ admit elementary cut-elimination, the same holds for $\mathbf{L} \mathbf{J}$.

This paper uses variants of CERES to obtain the above mentioned results. CERES [7] is a global semi-semantical cut-elimination method which subsumes the usual stepwise reduction methods and allows a better transformation of cutfree proofs due to its global nature.

\section{PRELIMINARIES}

We will describe now the exact setting in which we prove our results. We will represent classical proofs in the (multiplicative) calculus LK, depicted in Figure 1. Proofs will be considered intuitionistic if they are in the $\mathbf{L J}$ calculus, which is obtained by simply restricting $\mathbf{L K}$ to sequents with at most one formula on the right side. Consequently, LJ has no contraction right rule. We will use the term proof to denote a derivation from tautological axioms (i.e., $A \vdash A$ ) and use derivation otherwise.

Definition 1 ((Negative) sequent). A sequent is a structure $S: \quad \Gamma \vdash \Delta$ where $\Gamma$ and $\Delta$ are multisets of formulas. $S$ is called negative if $\Delta$ is empty.

Definition 2 (Polarity). Let $F$ be a formula and $F^{\prime}$ a subformula of $F$. Then we can define the polarity of $F^{\prime}$ in $F$, i.e., $F^{\prime}$ can be positive or negative in $F$, according to the following criteria:

- If $F \equiv F^{\prime}$, then $F^{\prime}$ is positive in $F$.

- If $F \equiv A \wedge B$ or $F \equiv A \vee B$ or $F \equiv \forall x$.A or $F \equiv \exists x$.A and $F^{\prime}$ is positive (negative) in $A$ or $B$, then $F^{\prime}$ is positive (negative) in $F$.

- If $F \equiv A \rightarrow B$ and $F^{\prime}$ is positive (negative) in $B$, then $F^{\prime}$ is positive (negative) in $F$.

- If $F \equiv A \rightarrow B$ and $F^{\prime}$ is positive (negative) in $A$, then $F^{\prime}$ is negative (positive) in $F$.

- If $F \equiv \neg A$ and $F^{\prime}$ is positive (negative) in $A$, then $F^{\prime}$ is negative (positive) in $F$.

Definition 3 (Strong and weak quantifiers). Let $F$ be $a$ formula. If $\forall x$ occurs positively (negatively) in $F$, then $\forall x$ is called a strong (weak) quantifier. If $\exists x$ occurs positively (negatively) in $F$, then $\exists x$ is called $a$ weak (strong) quantifier. Let $A_{1}, \ldots, A_{n} \vdash B_{1}, \ldots, B_{m}$ be a sequent. A quantifier is called strong (weak) in this sequent if it is strong (weak) in the corresponding formula $A_{1} \wedge \ldots \wedge A_{n} \rightarrow B_{1} \vee \ldots \vee B_{m}$.

Strong quantifiers in a sequent will be those introduced by the inferences $\forall_{r}$ and $\exists_{l}$.

Definition 4 (Skolemized sequent). A sequent is called skolemized if it contains no strong quantifiers.

In this paper we investigate proofs of skolemized negative sequents. Observe that, in this class of proofs, classical and intuitionistic provability coincide (which would not be the

$$
\begin{array}{cl}
\overline{A \vdash A} & \frac{\Gamma_{1} \vdash \Delta_{1}, P \quad \Gamma_{2}, P \vdash \Delta_{2}}{\Gamma_{1}, \Gamma_{2} \vdash \Delta_{1}, \Delta_{2}} c u t \\
\frac{\Gamma \vdash \Delta, P}{\Gamma, \neg P \vdash \Delta} \neg_{l} & \frac{\Gamma, P \vdash \Delta}{\Gamma \vdash \Delta, \neg P} \neg_{r} \\
\frac{P_{i}, \Gamma \vdash \Delta}{P_{1} \wedge P_{2}, \Gamma \vdash \Delta} \wedge_{l i} & \frac{\Gamma_{1} \vdash \Delta_{1}, P \quad \Gamma_{2} \vdash \Delta_{2}, Q}{\Gamma_{1}, \Gamma_{2} \vdash \Delta_{1}, \Delta_{2}, P \wedge Q} \wedge_{r} \\
\frac{P, \Gamma_{1} \vdash \Delta_{1} \quad Q, \Gamma_{2} \vdash \Delta_{2}}{P \vee Q, \Gamma_{1}, \Gamma_{2} \vdash \Delta_{1}, \Delta_{2}} \vee_{l} & \frac{\Gamma \vdash \Delta, P_{i}}{\Gamma \vdash \Delta, P_{1} \vee P_{2}} \vee_{r i} \\
\frac{\Gamma_{1} \vdash \Delta_{1}, P \quad Q, \Gamma_{2} \vdash \Delta_{2}}{P \rightarrow Q, \Gamma_{1}, \Gamma_{2} \vdash \Delta \Delta_{2}} \rightarrow_{l} & \frac{\Gamma, P \vdash \Delta, Q}{\Gamma \vdash \Delta, P \rightarrow Q} \rightarrow_{r} \\
\frac{P\{x \leftarrow \alpha\}, \Gamma \vdash \Delta}{\exists x . P, \Gamma \vdash \Delta} \exists_{l} & \frac{\Gamma \vdash \Delta, P\{x \leftarrow t\}}{\Gamma \vdash \Delta, \exists x . P} \exists_{r} \\
\frac{P\{x \leftarrow t\}, \Gamma \vdash \Delta}{\forall x . P, \Gamma \vdash \Delta} \forall_{l} & \frac{\Gamma \vdash \Delta, P\{x \leftarrow \alpha\}}{\Gamma \vdash \Delta, \forall x . P} \forall_{r} \\
\frac{P, P, \Gamma \vdash \Delta}{P, \Gamma \vdash \Delta} c_{l} & \frac{\Gamma \vdash \Delta, P, P}{\Gamma \vdash \Delta, P} c_{r} \\
\frac{\Gamma \vdash \Delta}{P, \Gamma \vdash \Delta} w_{l} & \frac{\Gamma \vdash \Delta}{\Gamma \vdash \Delta, P} w_{r}
\end{array}
$$

Figure 1. LK: Sequent calculus for classical logic. It is assumed that: $\alpha$ is a variable not contained in $P, \Gamma$ or $\Delta ; t$ does not contain variables bound in $P ; i \in\{1,2\}$; and $A$ is an atomic formula.

case if strong quantifiers in the end-sequents were allowed). Nevertheless, this does not mean that the classical and intuitionistic proofs of such sequents will be the same. In this paper, we establish a relation between them, namely, that they have about the same complexity. More precisely, we show that within this class there is an elementary transformation of classical cut-free proofs into intuitionistic cut-free proofs. This transformation is based on the cutelimination method CERES.

\section{CUt-elimination B Y NEGATIVE RESOlution}

This cut-elimination procedure for (a sub-class of) intuitionistic logic is a modification of the CERES method (cutelimination by resolution) for classical logic [7] and was first described in [8]. It can be split into the following steps:

Let $\varphi$ be an $\mathbf{L J}$-proof with cuts of a negative skolemized sequent $\Gamma \vdash$.

1) Extraction of the characteristic clause set $C L(\varphi)$.

2) Refutation of $C L(\varphi)$ using the negative refinement of resolution.

3) Extraction of a set of projections $\pi(C)$ for every $C \in$ $C L(\varphi)$

4) Merging of refutation and projections into a proof with only atomic cuts.

5) Left-shift cut-elimination of the atomic cuts.

The method uses the concept of formula ancestors, which we define now. 
Definition 5 (Formula ancestor). Let $\nu$ be a formula occurrence in a sequent calculus proof $\varphi$. If $\nu$ is a principal formula occurrence of an inference then the occurrences of the auxiliary formula (formulas) in the premises are ancestors of $\nu$. If $\nu$ is principal formula of a weakening or occurs in an axiom then $\nu$ has no ancestor. If $\nu$ is not a principal occurrence then the corresponding occurrences in contexts of the (premise) premises are ancestors of $\nu$. The ancestor relation is then defined as the reflexive transitive closure.

We will use the following proof as our running example to clarify each definition:

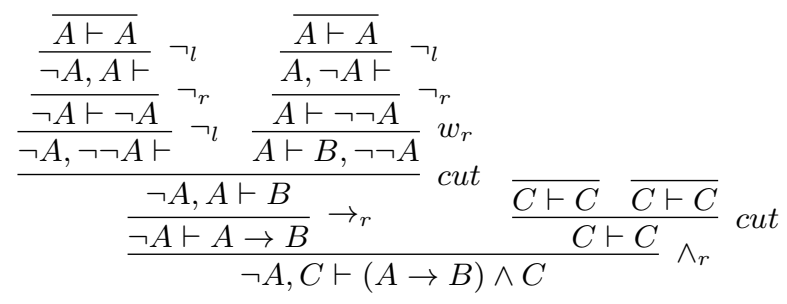

The clause set extraction consists in, intuitively, collecting all atomic ancestors of the cut formulas which occur in the axioms of the proof. The clauses are formed depending on how these atoms are related via binary inferences in the proof.

Definition 6 (Clause). A sequent $\Gamma \vdash \Delta$ is called a clause if $\Gamma$ and $\Delta$ are multisets of atoms.

Definition 7 (Characteristic clause-set). Let $\varphi$ be a proof of a skolemized sequent. The characteristic clause set is built recursively from the leaves of the proof until the end sequent. Let $\nu$ be a sequent in this proof. Then:

- If $\nu$ is an axiom, then $C L(\nu)$ contains the sub-sequent of $\nu$ composed only of cut ancestors.

- If $\nu$ is the result of the application of a unary rule on a sequent $\mu$, then $C L(\nu)=C L(\mu)$

- If $\nu$ is the result of the application of a binary rule on sequents $\mu_{1}$ and $\mu_{2}$, then we distinguish two cases:

- If the rule is applied to ancestors of the cut formula, then $C L(\nu)=C L\left(\mu_{1}\right) \cup C L\left(\mu_{2}\right)$

- If the rule is applied to ancestors of the end sequent, then $C L(\nu)=C L\left(\mu_{1}\right) \times C L\left(\mu_{2}\right)$

Where $^{1}: C L\left(\mu_{1}\right) \times C L\left(\mu_{2}\right)=\left\{C \circ D \mid C \in C L\left(\mu_{1}\right), D \in\right.$ $\left.C L\left(\mu_{2}\right)\right\}$

If $\nu_{0}$ is the root node $C L\left(\nu_{0}\right)$ is called the characteristic clause set of $\varphi$.

The clause set of our example proof contains one negative clause:

$$
C L(\varphi)=\{A \vdash C ; A, C \vdash ; \vdash A, C ; C \vdash A\}
$$

\footnotetext{
${ }^{1}$ The operation $\circ$ represents the merging of sequents, i.e., $(\Gamma \vdash \Delta) \circ\left(\Gamma^{\prime} \vdash\right.$ $\left.\Delta^{\prime}\right)=\Gamma, \Gamma^{\prime} \vdash \Delta, \Delta^{\prime}$.
}

The next step is to obtain a negative resolution refutation of $C L(\varphi)$. It is thus important to show that this set is always refutable.

Theorem 1. Let $\varphi$ be a proof of a skolemized end-sequent. Then the characteristic clause set $C L(\varphi)$ is refutable.

Proof: In [7], [9].

Definition 8 (Resolution calculus). The resolution calculus consists of the following rules:

$$
\frac{\Gamma \vdash \Delta, A \quad \Gamma^{\prime}, A^{\prime} \vdash \Delta^{\prime}}{\Gamma \sigma, \Gamma^{\prime} \sigma \vdash \Delta \sigma, \Delta^{\prime} \sigma} R \frac{\Gamma, A, A^{\prime} \vdash \Delta}{\Gamma \sigma, A \sigma \vdash \Delta \sigma} C_{l} \frac{\Gamma \vdash \Delta, A, A^{\prime}}{\Gamma \sigma \vdash \Delta \sigma, A \sigma} C_{r}
$$

Where $\sigma$ is the most general unifier of $A$ and $A^{\prime}$.

Definition 9 (Negative resolution refinement). A resolution derivation is called negative if, in every application of the rule $R$, one of the clauses in the premise is negative and the only factoring rule is $C_{l}$ applied to negative clauses, i.e. all rules are of the form:

$$
\frac{\Gamma \vdash \Delta, A \quad \Gamma^{\prime}, A^{\prime} \vdash}{\Gamma \sigma, \Gamma^{\prime} \sigma \vdash \Delta \sigma} R \frac{\Gamma, A, A^{\prime} \vdash}{\Gamma \sigma, A \sigma \vdash} C_{l}
$$

Theorem 2. The negative resolution refinement is complete.

Proof: By Theorem 3.6.1. in [10] and by sign renaming.

We give a negative resolution refutation of $C L(\varphi)$ :

$$
\frac{\frac{C \vdash A \quad A, C \vdash}{\frac{C, C \vdash}{C \vdash} c_{l}} R \quad A, C \vdash}{\frac{\vdash A \quad}{\frac{\vdash A}{\vdash}} \quad \frac{A \vdash C \quad A, C \vdash}{\frac{A, A \vdash}{A \vdash} c_{l}} R} R
$$

From Theorems 1 and 2 we conclude that there is always a negative resolution refutation of the clause set. But in order to use this refutation in our method, it needs to be grounded. To this aim we transform the refutation to tree form, rename the variables of clauses in the leaves and apply the global unifier of all the resolutions; finally all variables can be replaced by a constant symbol. For details see [9].

Since our running example is a propositional proof, the resolution refutation is already grounded.

Each clause in the clause set will have a projection associated with it. A projection of a clause $C$ is a derivation built from $\varphi$ by taking the axioms in which the atoms of $C$ occur and all the inferences that operate on end-sequent ancestors. As a result, the end-sequent of a projection will be the end-sequent of $\varphi$ plus the atoms of $C$.

Definition 10 (Projections). Let $\varphi$ be a proof and $\xi$ the last (lower most) inference with conclusion $\nu$. We define $p(\nu)$ as the set of projections $\{\pi(C) \mid C \in C L(\nu)\}$. Each projection $\pi(C)$ is a cut-free proof of the sequent $\nu \circ C$.

- If $\xi$ is an axiom, then $p(\nu)=\{\varphi\}$.

- If $\xi$ is a unary rule with premise $\mu$ : 
- If $\xi$ operates on a cut ancestor, then $p(\nu)=p(\mu)$.

- If $\xi$ operates on an end-sequent ancestor, then $p(\nu)$ is the set of:

$$
\frac{\pi\left(C_{i}\right)}{\zeta} \xi
$$

such that $\pi\left(C_{i}\right) \in p(\mu)$.

- If $\xi$ is a binary rule with premises $\mu_{1}$ and $\mu_{2}$.

- If $\xi$ operates on a cut ancestor, then $p(\nu)=$ $p\left(\mu_{1}\right) \cup p\left(\mu_{2}\right)$.

- If $\xi$ operates on an end-sequent ancestor, then $p(\nu)$ is the set of:

$$
\frac{\pi\left(C_{i}^{1}\right) \pi\left(C_{j}^{2}\right)}{\zeta} \xi
$$

such that $\pi\left(C_{i}^{1}\right) \in p\left(\mu_{1}\right)$ and $\pi\left(C_{j}^{2}\right) \in p\left(\mu_{2}\right)$.

In each step, it might be necessary to weaken the auxiliary formulas of an inference. Moreover, if not all formulas of the end-sequent are present after constructing the projection, they are weakened as well.

Note that no rule operates on cut ancestors, therefore they occur as atoms in the end-sequent of the projections.

As an example, here is the projection of the clause $\vdash A, C$ (where these atoms are marked with $\star$ ):

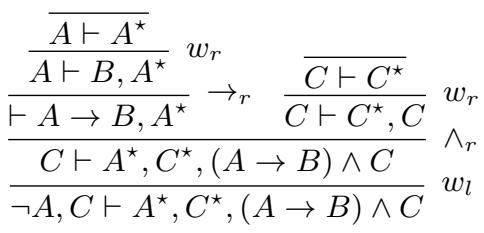

Observe that, although the input proof was intuitionistic, the projections obtained from it might be classical. But projections of negative clauses are always valid intuitionistic derivations.

Theorem 3. Let $\varphi$ be an $\mathbf{L J}$-proof. Then the projections of negative clauses are valid $\mathbf{L} \boldsymbol{J}$ proofs.

Proof: The projections are obtained by applying inferences from $\varphi$ that operate on end-sequent ancestors. Since this is an LJ -proof, these are initially valid intuitionistic inferences. The only thing that changes on the projections' sequents (to which the inferences are applied) is the possible extra atoms from the clause. Given the single conclusion restriction of $\mathbf{L} \mathbf{J}$, the only time this is violated is when atoms occur on the right side of the sequent. As this is not the case for negative clauses, the rules in the projections of such clauses will be single conclusion and therefore the projection itself will be a valid $\mathbf{L J}$ proof.

Given the projections and a grounded negative resolution refutation, it is possible to build a proof $\hat{\varphi}$ of $\Gamma \vdash$ with only atomic cuts.
Definition 11 (Context product). Let $C$ be a sequent and $\varphi$ be an $\mathbf{L K}$ derivation with end-sequent $S$ such that no free variable in $C$ occurs as eigenvariable in $\varphi$. We define the context product $C \star \varphi$ (which gives a derivation of $C \circ S$ ) inductively:

- If $\varphi$ consists only of an axiom, then $C \star \varphi$ is composed by one sequent: $C \circ S$.

- If $\varphi$ ends with a unary rule $\xi$ :

$$
\begin{aligned}
& \varphi^{\prime} \\
& \frac{S^{\prime}}{S} \xi
\end{aligned}
$$

then we assume that $C \star \varphi^{\prime}$ is already defined and thus $C \star \varphi$ is:

$$
\begin{aligned}
& C \star \varphi^{\prime} \\
& \frac{C \circ S^{\prime}}{C \circ S} \xi
\end{aligned}
$$

Since $C$ does not contain free variables which are eigenvariables of $\varphi$, the context product is well defined also if $\xi$ is $\forall_{r}$ or $\exists_{l}$, although this case does not occur in our setting.

- If $\varphi$ ends with a binary rule $\xi$ :

$$
\begin{array}{ll}
\varphi_{1} & \varphi_{2} \\
S_{1} & S_{2} \\
\hline & S
\end{array}
$$

then assume that $C \star \varphi_{1}$ and $C \star \varphi_{2}$ are already defined. We define $C \star \varphi$ :

$$
\begin{aligned}
& C \star \varphi_{1} \quad C \star \varphi_{2} \\
& \frac{C \circ S_{1} \quad C \circ S_{2}}{\frac{C \circ C \circ S}{C \circ S} c^{*}} \xi
\end{aligned}
$$

Note that since the formulas in $C$ will come from both branches, and we are working in a multiplicative calculus, after applying a binary rule we need to contract the formulas from $C$ to obtain the correct multi-set.

Definition 12 (Negative CERES normal form). Let $\varphi$ be an $\boldsymbol{L} \boldsymbol{J}$ proof of a negative skolemized sequent $S, C L(\varphi)$ its clause set and $\varrho$ a grounded negative resolution refutation of $C L(\varphi)$. We first construct $\varrho^{\prime}=S \star \varrho$. Note that this is a derivation of $S$ from a set of axioms $C \circ S$, with $C \in C L(\varphi)$, which are exactly the end-sequents of the projections $\pi(C)$ of $\varphi$. Now we define $\varphi(\varrho)$ by replacing all axioms of $\varrho^{\prime}$ by the respective projections. By definition, $\varphi(\varrho)$ is an $\mathbf{L K}$ proof of $S$ with only atomic cuts. We call it the negative CERES normal form of $\varphi$ with respect to $\varrho$.

This procedure of obtaining a negative CERES normal form from an $\mathbf{L J}$ proof $\varphi$ is called negative CERES and we will denote the proof with atomic cuts obtained by $\hat{\varphi}$. The only modification of negative CERES over the CERES method is the enforcement of negative resolution.

Since we are using negative resolution and the endsequent of $\varphi$ is negative, every atomic cut in $\hat{\varphi}$ will have the shape: 


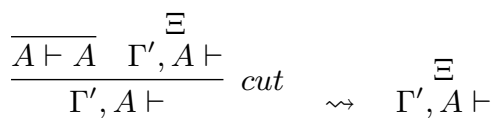

Figure 3. Elimination of the cut in left-shift cut-elimination.

$$
\frac{\Gamma \vdash \Delta, A \quad A, \Gamma^{\prime} \vdash}{\Gamma, \Gamma^{\prime} \vdash \Delta} c u t
$$

Note that, since projections might be classical proofs, $\hat{\varphi}$ may also be a classical proof. Nevertheless it can be transformed again into an intuitionistic proof by removing the atomic cuts.

Since the original proof was in a single conclusion calculus, we know that every sequent with more than one formula on the right side must contain at most one end-sequent ancestor, the other formulas being atomic cut-ancestors. Therefore, if we can eliminate the atomic cuts maintaining always at most one end-sequent ancestor on the right side of every sequent, we will obtain an $\mathbf{L J}$ proof. Now we show how to achieve this by insisting on a specific discipline for reductive cut-elimination.

Definition 13 (Left-shift cut-elimination). Let $\hat{\varphi}$ be a proof with only atomic cuts. We call left-shift cut-elimination the process of removing the atomic cuts that, starting from the top most cuts down, (1) permutes the cut over all the rules of its left branch (Figure 2) until reaching an axiom and (2) eliminates the cut by using the proof on its right branch (Figure 3).

Theorem 4. Let $\varphi$ be an LJ-proof with cuts and $\hat{\varphi}$ the negative CERES normal form obtained with negative CERES. Then eliminating the cuts from $\hat{\varphi}$ using left-shift cut-elimination yields an $\mathbf{L J}$-proof.

Proof: Although $\varphi$ is an $\mathbf{L J}$ proof, each inference $\rho$ in $\hat{\varphi}$ might be applied to a multiple conclusion sequent because of atomic cut-ancestors. By reductively eliminating the cuts, we make sure that the resulting proof's sequents contain no atomic cut-ancestors on the right, but there is no guarantee that they will all be single conclusion. This can be ensured by two things: (1) $\varphi$ is a proof of a negative sequent and (2) left-shift cut-elimination is used to eliminate the atomic cuts from $\hat{\varphi}$.

Let $\rho$ be an inference in $\hat{\varphi}$ that was an instance of an inference in $\varphi$ (which was originally applied to a single conclusion sequent). We have thus to show that after leftshift cut-elimination, every $\rho$ will be applied to a single conclusion sequent.

First note that every inference $\rho$ is applied to a sequent such that its right context contain at most one end-sequent ancestor, the other formulas being atomic cut ancestors. Now observe that in the reduction rules of Figure 2, the $\rho$ in the resulting derivation is always applied to a sequent whose right context contains strictly less formulas then in the original derivation. Moreover, these are all the rules necessary for eliminating the atomic cuts, as there is no right contraction of the cut-formulas because there is no right contraction in the negative resolution fragment. After eliminating all the cuts, every $\rho$ will be applied to a sequent whose right context contains at most one end-sequent ancestor and no cut ancestors, exactly as it was in $\varphi$.

Second, upon actually eliminating the cut (Figure 3), the derivation used is a negative projection which, by Theorem 3, is an $\mathbf{L J}$ proof.

The final proof is therefore a valid LJ proof.

\section{TRANSFORMING CLASSICAL INTO INTUITIONISTIC PROOFS}

A. The double negation translation for the weak quantifier fragment

As (quantified) cuts always contain quantifiers in both polarities, we have to translate cut-free $\mathbf{L K}$ derivations.

Definition 14 (Left-sided intuitionistic translation). Let $\varphi$ be a cut-free $\mathbf{L K}$ proof containing only weak quantifiers with end-sequent $\Gamma \vdash \Delta$. We define the left-sided intuitionistic translation $I(\varphi)$ an $\mathbf{L} \boldsymbol{J}$ proof with cuts and endsequent $\neg \neg \Gamma, \neg \Delta \vdash$ (where $\neg \neg \Gamma$ and $\neg \Delta$ denote the sets $\{\neg \neg F \mid F \in \Gamma\}$ and $\{\neg F \mid F \in \Delta\}$, respectively) inductively on $\varphi$.

- If $\varphi$ consists of only one axiom $C \vdash C$, then $I(\varphi)$ is the $\mathbf{L} \boldsymbol{J}$ derivation:

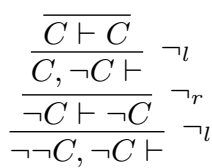

- If $\varphi$ ends with a structural rule, then we can assume, by induction, that $I\left(\varphi^{\prime}\right)$ is already defined and $I(\varphi)$ is straightforward:

$$
\begin{aligned}
& \frac{\varphi^{\prime}}{\Gamma, C, C \vdash \Delta} c_{l} \Rightarrow \frac{I\left(\varphi^{\prime}\right)}{\neg \vdash \Gamma, \neg \neg C, \neg \neg C, \neg \Delta \vdash} c_{l} \\
& \varphi^{\prime} \quad I\left(\varphi^{\prime}\right) \\
& \frac{\Gamma \vdash \Delta, C, C}{\Gamma \vdash \Delta, C} c_{r} \Rightarrow \frac{\neg \neg \Gamma, \neg \Delta, \neg C, \neg C \vdash}{\neg \neg \Gamma, \neg \Delta, \neg C \vdash} c_{l} \\
& \frac{\varphi^{\prime}}{\Gamma \vdash \Delta} \underset{\vdash, C \vdash \Delta}{ } w_{l} \Rightarrow \frac{\neg \neg \Gamma, \neg \Delta \vdash}{\neg \neg \Gamma, \neg \neg C, \neg \Delta \vdash} w_{l} \\
& \begin{array}{c}
\varphi^{\prime} \\
\Gamma \vdash \Delta \\
\Gamma \vdash \Delta, C
\end{array} w_{r} \Rightarrow \frac{I\left(\varphi^{\prime}\right)}{\neg \neg \Gamma, \neg, \neg \Delta, \neg C \vdash} w_{l}
\end{aligned}
$$




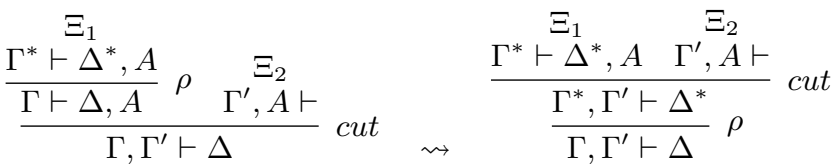

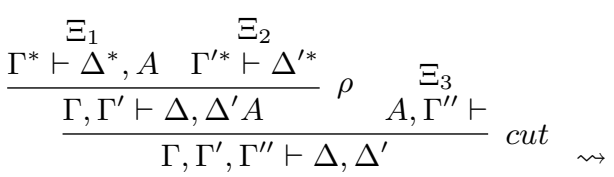

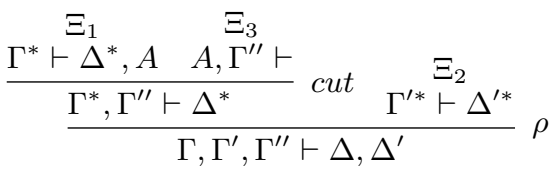

$$
\begin{aligned}
& \Xi_{1} \quad \Xi_{2} \\
& \frac{\Gamma^{*} \vdash \Delta^{*} \quad \Gamma^{* *} \vdash \Delta^{\prime *}, A}{\Gamma, \Gamma^{\prime} \vdash \Delta, \Delta^{\prime} A} \rho \quad \begin{array}{l}
\Xi_{3} \\
\hline, \Gamma^{\prime \prime} \vdash
\end{array} \\
& \Gamma, \Gamma^{\prime}, \Gamma^{\prime \prime} \vdash \Delta, \Delta^{\prime} \quad c u t \\
& \underset{\Gamma^{*} \vdash \Delta^{*}}{\Xi_{1} \quad \frac{\Gamma^{* *} \vdash \Delta^{\prime *}, A \quad A, \Gamma^{\prime \prime} \vdash}{\Gamma^{*}, \Gamma^{\prime \prime} \vdash \Delta^{\prime *}}} \text { cut }
\end{aligned}
$$

Figure 2. Reduction rules for left-shift cut-elimination.

- If $\varphi$ ends with a logical rule, we distinguish the cases in Figure 4 (in all cases we assume, by induction, that $I\left(\varphi^{\prime}\right)$ and $I\left(\varphi^{\prime \prime}\right)$ are defined $)$.

Definition 15. Let $\varphi$ be an $\mathbf{L K}$ or $\boldsymbol{L} \boldsymbol{J}$ proof. We define the size of $\varphi$, denoted by $\|\varphi\|$, as the number of symbol occurrences in the proof.

Proposition 1. Let $\varphi_{K}$ be a cut-free $\mathbf{L K}$ derivation of $\Gamma \vdash B$. Then it can be translated into an $\mathbf{L J}$ derivation with cuts of $\neg \neg \Gamma \vdash \neg \neg B$, denoted by $\varphi_{I}$, such that $\left\|\varphi_{I}\right\| \leq\left\|\varphi_{K}\right\|^{2}$.

Proof: The left-sided intuitionistic translation of $\varphi_{K}$, gives us an intuitionistic proof $I\left(\varphi_{K}\right)$ with end-sequent $\neg \neg \Gamma, \neg B \vdash$. By simply applying a $\neg_{r}$ inference to the endsequent of this proof, we obtain an $\mathbf{L J}$ derivation with cuts of $\neg \neg \Gamma \vdash \neg \neg B$. The number of inserted proofs in $I\left(\varphi_{K}\right)$ (indicated by $\nabla$ ) is linear in the number of inferences in $\varphi_{K}$ and so is their size.

Note that not much is known about the complexity of the elimination of intuitionistic cuts with quantifiers of the same polarity and arbitrary propositional structure. Therefore the proof transformation given in Proposition 1 does not give us an elementary bound on the mapping of cut-free LK to cut-free $\mathbf{L} \mathbf{J}$ proofs within this class of proofs.

\section{B. An elementary translation of cut-free proofs}

Definition 16. Let $\varrho$ be a resolution refutation. We define the size of $\varrho$, denoted by $\|\varrho\|$, as the number of symbol occurrences in $\varrho$.

Theorem 5. There exists an elementary function $h$ s.t., given a proof $\varphi$, a resolution refutation $\varrho$ of $C L(\varphi)$ and a corresponding CERES normal form $\hat{\varphi}$, we have $\|\hat{\varphi}\| \leq$ $h(\|\varphi\|,\|\varrho\|)$.

Proof: In [9] Lemma 6.5.3 it is shown that there is a strictly increasing elementary function $H$ s.t., given a proof $\varphi$ and a resolution refutation $\varrho$ of $C L(\varphi),\left\|\varrho^{\prime}\right\| \leq$
$H(\|\varphi\|, l(\varrho))$ for an appropriate ground resolution refutation $\varrho^{\prime}$ of $C L(\varphi)$ (where $l(\varrho)$ is the number of proof nodes in $\varrho)$. As $l(\varrho) \leq\|\varrho\|$ we get

$$
\text { (1) }\left\|\varrho^{\prime}\right\| \leq H(\|\varphi\|,\|\varrho\|) \text {. }
$$

By Lemma 6.5.4 in [9] we have

$$
(2)\|\hat{\varphi}\| \leq H(\|\varphi\|, l(\varrho)) *\|\varphi\| * r\left(\varrho^{\prime}\right)
$$

for the CERES normal form $\hat{\varphi}$ corresponding to $\varrho$, where $r\left(\varrho^{\prime}\right)$ is the size of a maximal term occurring in $\varrho^{\prime}$. Clearly $r\left(\varrho^{\prime}\right) \leq\left\|\varrho^{\prime}\right\|$. Therefore, putting (1) and (2) together we obtain

$$
\|\hat{\varphi}\| \leq H(\|\varphi\|,\|\varrho\|)^{2} *\|\varphi\| .
$$

In defining $h(x, y)=H(x, y)^{2} * x$ ( $h$ is clearly elementary as $H$ is) we eventually obtain

$$
\|\hat{\varphi}\| \leq h(\|\varphi\|,\|\varrho\|)
$$

Theorem 6. Let $\hat{\varphi}$ be a negative CERES normal form of $\varphi$ and let $\varphi_{0}$ be the cut-free proof obtained after applying left-shift cut-elimination to $\hat{\varphi}$. Then there exists a constant $c$ (independent of $\varphi$ ) such that $\left\|\varphi_{0}\right\| \leq c *\|\hat{\varphi}\|$.

Proof: Given the transformations in Figures 2 and 3 (which are all the rules necessary for eliminating the atomic cuts), observe that the right-hand side uses only those derivations that were already present on the left, without duplicates. Thus left-shift cut-elimination does not increase the number of inference nodes in the proof. As the transformation in Figure 3 even eliminates an inference, $\varphi_{0}$ contains less inferences than $\hat{\varphi}$, provided there is at least one cut in $\hat{\varphi}$. Still the rules in Figure 2 may mildly increase the symbolic size of a proof. Note that e.g. in the first rule we may have $\left\|\Gamma^{*}\right\|>\|\Gamma\|$ ( $\rho$ may be $\forall_{l}$ and a large term is eliminated (top-down) by the rule which now occurs twice in the result). But this increase happens for every rule 


$$
\begin{aligned}
& \varphi^{\prime} \\
& \frac{\Gamma \vdash \Delta, C}{\Gamma, \neg C \vdash \Delta} \neg l \quad \rightsquigarrow
\end{aligned}
$$

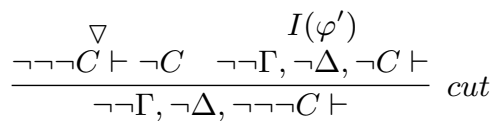

$$
\begin{aligned}
& \varphi^{\prime} \\
& \Gamma, C \vdash \Delta \neg_{r} \quad I\left(\varphi^{\prime}\right) \\
& \frac{\Gamma, C \vdash \Delta}{\Gamma \vdash \Delta, \neg C} \neg r \quad \rightsquigarrow \quad \neg \neg \Gamma, \neg \neg C, \neg \Delta \vdash
\end{aligned}
$$

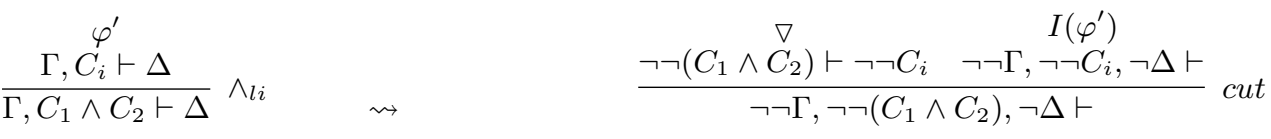

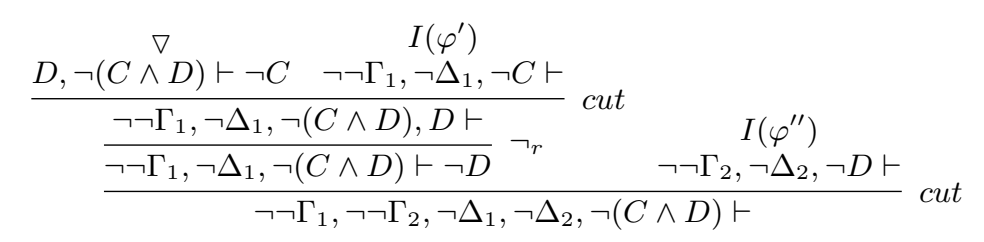

$$
\begin{aligned}
& \frac{\Gamma_{1} \vdash \Delta_{1}, C \quad \Gamma_{2} \vdash \Delta_{2}, D}{\Gamma_{1}, \Gamma_{2} \vdash \Delta_{1}, \Delta_{2}, C \wedge D} \wedge_{r} \rightsquigarrow
\end{aligned}
$$

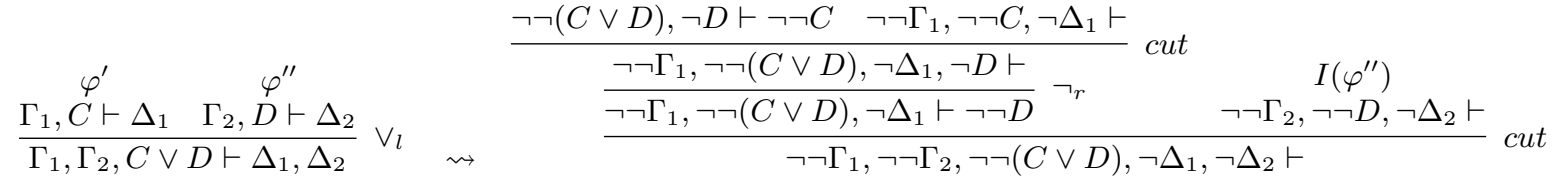

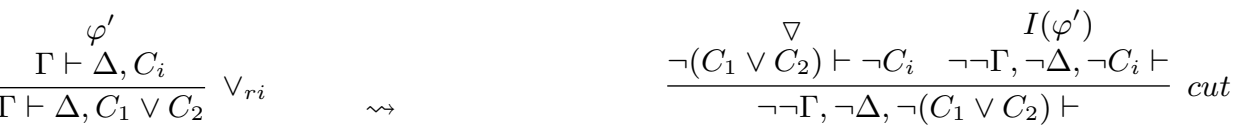

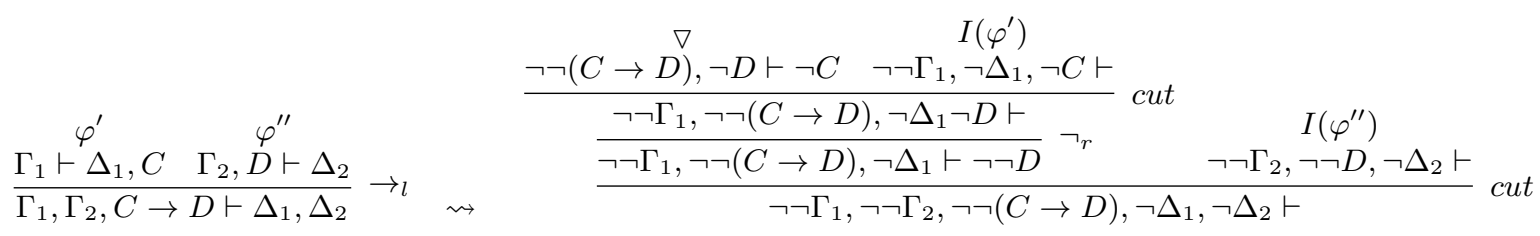

$$
\begin{aligned}
& \frac{\varphi^{\prime}}{\Gamma, C \vdash \Delta, D} \underset{\vdash \vdash \Delta, C \rightarrow D}{\Gamma \vdash r} \rightsquigarrow \\
& \frac{\neg\left(C \rightarrow \stackrel{\nabla}{D} \vdash \neg \neg C \quad \frac{\nabla}{\neg(C \rightarrow D) \vdash \neg D \quad \neg \neg \Gamma, \neg \neg C, \neg \Delta, \neg D \vdash}\right.}{\neg} c u t
\end{aligned}
$$

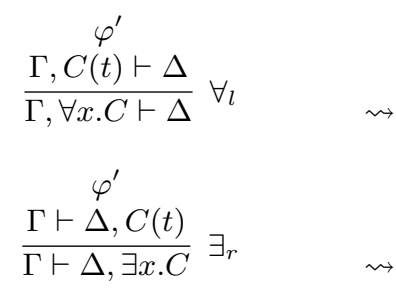

$$
\begin{aligned}
& \frac{\neg \neg(\forall x . C) \vdash \neg \neg C(t) \quad \neg \neg \Gamma, \neg \neg C(t), \neg \Delta \vdash}{\neg \neg \Gamma, \neg \neg(\forall x . C), \neg \Delta \vdash} c u t \\
& \frac{\neg(\exists x . C) \vdash \neg C(t) \quad \neg \neg \Gamma, \neg \Delta, \neg C(t) \vdash}{\neg \neg \Gamma, \neg \Delta, \neg(\exists x . C) \vdash} c u t
\end{aligned}
$$

Figure 4. The transformation $I(\varphi)$ for logical connectives. In all the derivations, $\nabla$ indicate an easy intuitionistic proof. 
$\rho$ (coming from a left-hand-side of a cut) only once, and the material causing the increase is already present in the original proof. Therefore, there exists a constant $c$ such that: $\left\|\varphi_{0}\right\| \leq c *\|\hat{\varphi}\|$.

Remark 1. The worst-case complexity for the elimination of atomic cuts is exponential in general. In fact, given a CERES normal form based on an arbitrary resolution refutation, cutelimination may lead to an exponential increase in size. That cut-elimination is linear for negative CERES normal forms is due to the fact that there are no right contractions on the atoms of the cuts and proof duplication can be avoided. The price to pay is that negative CERES normal forms may be exponential in the minimal size of CERES normal forms.

Proposition 2. Let $\varphi$ be a proof of a skolemized sequent $S$. Then $\|C L(\varphi)\| \leq 2^{\|\varphi\|}$.

Proof: In [9].

In [11] a comparison of reductive cut-elimination and CERES was given. It turned out that reductive cutelimination is, in some sense, redundant w.r.t. CERES. The measure of redundancy is the well known subsumption principle from automated deduction.

Let $\Gamma$ be a multiset of formulas; by $\operatorname{set}(\Gamma)$ we describe the set defined by the elements in $\Gamma$.

Definition 17 (subsumption). Let $C: \Gamma \vdash \Delta$ and $D: \Pi \vdash$ $\Lambda$ be clauses. We define $C \subseteq D$ if $\operatorname{set}(\Gamma) \subseteq \operatorname{set}(\Pi)$ and $\operatorname{set}(\Delta) \subseteq \operatorname{set}(\Lambda)$. We define $C \leq_{\text {sub }} D$ if there exists a substitution $\vartheta$ s.t. $C \vartheta \subseteq D$. Let $\mathcal{C}, \mathcal{D}$ be sets of clauses; then $\mathcal{C} \leq_{\text {sub }} \mathcal{D}$ if for every clause $D \in \mathcal{D}$ there exists a $C \in \mathcal{C}$ s.t. $C \leq_{\text {sub }} D$.

Proposition 3. Let $\varphi$ be a proof of a skolemized sequent $S$ and $\psi$ be a proof obtained from $\varphi$ via (one or more) cut-elimination steps of Gentzen's reductive method (without eliminating atomic cuts). Then $C L(\varphi) \leq_{\text {sub }} C L(\psi)$.

Proof: In [9], [11].

The subsumption principle can be extended from sets of clauses to resolution deductions: let us assume that $\mathcal{C}$ and $\mathcal{D}$ are sets of clauses s.t. $\mathcal{C} \leq_{\text {sub }} \mathcal{D}, \mathcal{D}$ is unsatisfiable and $\delta$ a resolution refutation of $\mathcal{D}$. Then there exists a resolution refutation $\gamma$ of $\mathcal{C}$ which "subsumes" $\delta . \gamma$ is in fact smaller than $\delta$, i.e. $\|\gamma\| \leq\|\delta\|$. For a formal definition of subsumption among resolution derivations see Definition 6.6.4 in [9]. The subsumption property of resolution refutations will be used in the proof of Theorem 7.

Definition 18. Let $F$ be a formula. We define the size of $F$, denoted by $\|F\|$, as the number of symbol occurrences in $F$.

Theorem 7. There exists an elementary function $g$ such that for every classical cut-free proof $\varphi$ of a skolemized sequent $S: \Gamma \vdash$ there exists an intuitionistic cut-free proof $\psi$ of $S$ such that $\|\psi\| \leq g(\|\varphi\|)$.
Proof: Let us consider a cut-free (classical) proof $\varphi$ of the skolemized sequent $S: A_{1}, \ldots, A_{n} \vdash$ (i.e. there are no strong quantifiers in the $A_{i}$ and - in classical logic the $A_{i}$ are equivalent to $\forall$-prenex forms). Now consider our transformation $T(\varphi)$ :

$$
\begin{aligned}
& \frac{A_{1}, \ldots, A_{n} \vdash}{\frac{A \vdash}{\vdash \neg A} \neg: r \quad l^{*} \quad \neg A, A_{1}, \ldots, A_{n} \vdash} \\
& \frac{A_{1}, \ldots, A_{n} \vdash}{}
\end{aligned}
$$

where $A=A_{1} \wedge \cdots \wedge A_{n}$ and $\psi$ is a cut-free intuitionistic proof of length polynomial in $\|A\| . T(\varphi)$ proves the same end-sequent as $\varphi$. Now observe that the cut-formula $\neg A$ on the left branch of the cut has only weak quantifiers, and only strong quantifiers on the right branch. Therefore, in classical logic, the cut-formula is equivalent to a $\Sigma_{1}$ formula and the cut has the strength of a $\Sigma_{1}$-cut only. Even without this detour over prenexing, it is clear that the cutelimination on cut $\neg A$ is elementary (in fact it is at most double exponential) by just following the Gentzen reduction of the quantifiers. Moreover it will suffice to eliminate the quantifiers in the cuts only. So let $\chi$ be the proof $T(\varphi)$ after elimination of the quantifiers in the cuts (so $\chi$ is a proof with quantifier-free cuts only). Putting things together there exists an elementary function $h$ s.t. $\|\chi\| \leq h(\|T(\varphi)\|)$; so there exists also an elementary function $f$ with $\|\chi\| \leq f(\|\varphi\|)$.

Now negative CERES comes into play: consider the characteristic clause set $C L(T(\varphi))$ and let $\mathcal{C}^{\prime}$ be the characteristic clause set of $\chi$. Note that $\mathcal{C}^{\prime}$ is a set of ground clauses (indeed we may assume that in a proof containing no strong quantifiers only ground terms are introduced by the quantifier rules). As $\chi$ is elementary in $T(\varphi), \mathcal{C}^{\prime}$ is as well by Proposition 2. By Proposition 3, the main subsumption result about reductive cut-elimination, $C L(T(\varphi))$ subsumes $\mathcal{C}^{\prime}$ and every resolution refutation $\varrho^{\prime}$ of $\mathcal{C}^{\prime}$ is subsumed by a resolution refutation $\varrho$ of $C L(T(\varphi))$. As $\mathcal{C}^{\prime}$ is ground, the length of a shortest negative resolution refutation $\varrho^{\prime}$ is at most exponential in $\left\|\mathcal{C}^{\prime}\right\|$ (note that the number of different negative clauses definable over the ground atoms is at most exponential). Moreover, $\|\varrho\| \leq\left\|\varrho^{\prime}\right\|$ (and $\varrho$ is also a negative resolution refutation as negative clauses can only be subsumed by negative clauses or by the empty clause). Clearly $\varrho^{\prime}$ is elementary in $T(\varphi)$ and so is $\varrho$, i.e. $\|\varrho\| \leq g(\|T(\varphi)\|)$ for an appropriate elementary function $g$ (independent of $\varrho$ and $\varphi)$. So we refute $C L(T(\varphi))$ with $\varrho$ and get a CERES-normal form $\hat{\varphi}$. By Theorem 5 we obtain $\|\hat{\varphi}\| \leq$ $h(\|T(\varphi)\|,\|\varrho\|)$ and thus $\|\hat{\varphi}\| \leq h(\|T(\varphi)\|, g(\|T(\varphi)\|)$. Define $h^{\prime}$ as $h^{\prime}(x)=h(x, g(x))$; then $h^{\prime}$ is elementary and

$$
\|\hat{\varphi}\| \leq h^{\prime}(\|T(\varphi)\|) \text {. }
$$

As $\varrho^{\prime}$ is the shortest negative resolution refutation of of $\mathcal{C}^{\prime}$ there are no tautological clauses occurring in $\varrho^{\prime}$ (note that a shortest negative resolution refutation never contains 
tautologies!). As a consequence also $\varrho$ does not contain tautological clauses. Now consider the proof $T(\varphi)$. As all inferences in $\varphi$ (within $T(\varphi)$ ) go into the cut formula $\neg A$, the clauses of the characteristic clause sets coming from $\varphi$ are all tautologies. But these tautologies are not used in $\varrho$. It follows that all projections in the CERES normal form of $T(\varphi)$, denoted here by $\hat{\varphi}$, come from the intuitionistic part of the proof. But note that, in this case, $\hat{\varphi}$ can be transformed into an intuitionistic cut-free proof $\psi$ via the method described in Theorem 4. By Theorem 6 also this transformation is elementary and $\|\psi\| \leq g(\|\varphi\|)$, where $g$ is an appropriate elementary function put together by the bound functions above.

We illustrate the transformation of Theorem 7 with an example. Let $\varphi$ be the $\mathbf{L K}$ proof:

$$
\begin{aligned}
& \frac{\overline{P f a \vdash P f a}}{\overline{P a, P f a \vdash P f a, P f f a}} w \\
& \overline{P a \vdash P f a, P f a \rightarrow P f f a} \rightarrow_{r} \\
& \frac{P}{\vdash P a \rightarrow P f a, P f a \rightarrow P f f a} \rightarrow r
\end{aligned}
$$

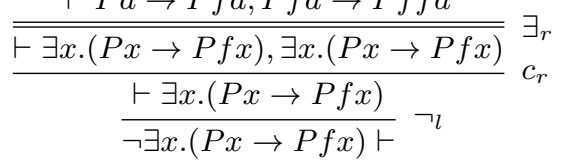

Then we can construct $\Xi=T(\varphi)$, which proves the same end-sequent but has a full intuitionistic proof on the right branch of the cut:

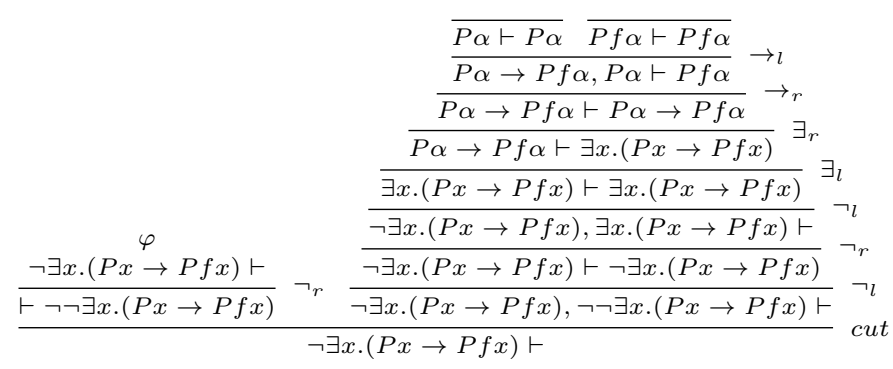

We apply the negative CERES method to this proof. The clause set extracted is the following:

$$
C L(\Xi)=\{P f a \vdash P f a ; \vdash P \alpha ; P f \alpha \vdash\}
$$

Note that the tautological clause $P f a \vdash P f a$, which came from the classical part of $\Xi$ can be eliminated. The only possible (negative) refutation is $\varrho$ :

$$
\frac{\frac{\vdash P \alpha}{\vdash P f a} \alpha \leftarrow f a \quad \frac{P f \alpha \vdash}{P f a \vdash}}{\vdash} R \leftarrow a
$$

Since $\varrho$ uses clauses that come from the intuitionistic side of $\Xi$, these are the only projections we need:

$$
\begin{aligned}
& \pi(\vdash P \alpha): \\
& \overline{P \alpha \vdash P \alpha} \\
& \frac{P \alpha \vdash P \alpha, P f \alpha}{P \alpha \vdash} w_{r} \\
& \frac{P P \alpha, P \alpha \rightarrow P f \alpha}{\vdash P}
\end{aligned}
$$

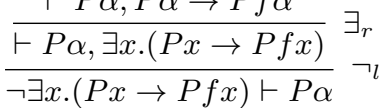

$$
\begin{aligned}
& \pi(P f \alpha \vdash): \\
& \frac{\overline{P f \alpha \vdash P f \alpha}}{P f \alpha, P \alpha \vdash P f \alpha} w_{l} \\
& \frac{\frac{P f \alpha, P \alpha \vdash P f \alpha}{P f \alpha \vdash P \alpha \rightarrow P f \alpha}}{P f \alpha \vdash \exists x .(P x \rightarrow P f x)} \rightarrow_{r} \\
& \frac{P f \alpha, \neg \exists x .(P x \rightarrow P f x) \vdash}{P l}
\end{aligned}
$$

Note that the projection of the negative clause is intuitionistic, but the other one is classical. Then we can compute the CERES normal form $\hat{\varphi}$ :

$$
\begin{aligned}
& \frac{\overline{P f a \vdash P f a}}{\overline{P f a \vdash P f a, P f f a}} w_{r} \quad \frac{\overline{P f a \vdash P f a}}{\rightarrow_{r}} w_{l} \\
& \overline{\vdash P f a, P f a \rightarrow P f f a} \rightarrow_{r} \quad \frac{P f a \vdash P a \rightarrow P f a}{P f} \rightarrow_{r} \\
& \frac{\overline{\vdash P f a, \exists x .(P x \rightarrow P f x)}}{\neg \exists x .(P x \rightarrow P f x) \vdash P f a} \neg_{r} \quad \frac{\overline{P f a \vdash \exists x .(P x \rightarrow P f x)}}{P f a, \neg \exists x .(P x \rightarrow P f x)} \neg_{r} \\
& \frac{\overline{\neg \exists x .(P x \rightarrow P f x) \vdash P f a} \neg l \quad \frac{P f a, \neg \exists x .(P x \rightarrow P f x) \vdash}{P l}}{\frac{\neg \exists x .(P x \rightarrow P f x), \neg \exists x .(P x \rightarrow P f x) \vdash}{\neg} c_{l}} \text { cut } \\
& \neg \exists x .(P x \rightarrow P f x) \vdash
\end{aligned}
$$

By performing left-shift cut-elimination, we obtain the $\mathbf{L} \mathbf{J}$ proof $\psi$ :

$$
\begin{gathered}
\frac{\overline{P f a \vdash P f a}}{P f a, P a \vdash P f a} w_{l} \\
\frac{\frac{P f a \vdash P a \rightarrow P f a}{P f a \vdash \exists x \cdot(P x \rightarrow P f x)}}{\frac{\neg \exists x .(P x \rightarrow P f x), P f a \vdash}{r}} \neg_{r} \\
\frac{\frac{\neg \exists x .(P x \rightarrow P f x), P f a \vdash P f f a}{\neg \exists x .(P x \rightarrow P f x) \vdash P f a \rightarrow P f f a}}{\frac{\neg \exists x .(P x \rightarrow P f x) \vdash \exists x .(P x \rightarrow P f x)}{\neg \exists .(P x \rightarrow P f x), \neg \exists x .(P x \rightarrow P f x)}} \rightarrow_{r} \\
\frac{\exists_{r}}{\neg \exists x .(P x \rightarrow P f x) \vdash} c_{l}
\end{gathered}
$$

The complexity of this transformation, according to the description of Theorem 7, has an exponential tower of three. We believe nevertheless that this bound is not sharp and that a double exponential bound exists, though not easy to prove.

Corollary 1. There exists an elementary function $h$ with the following property: given a classical cut-free proof $\varphi$ 


$$
\begin{aligned}
\Psi(\neg A) \equiv & \top \text { if } \neg A \text { is a substitution instance of a tautology } \\
& \neg \neg \neg \Psi(A) \text { otherwise }, \\
\Psi(A \circ B) \equiv & \top \text { if } A \circ B \text { is a substitution instance of a tautology } \\
& \neg \neg(\Psi(A) \circ \Psi(B)) \text { otherwise, where } \circ \in\{\wedge, \vee, \rightarrow\}, \\
\Psi(Q x . A(x)) \equiv & \neg \neg Q x . \Psi(A(x)) \text { for } Q \in\{\forall, \exists\}, \\
\Psi(A) \equiv & \neg \neg A \text { if } A \text { is atomic. }
\end{aligned}
$$

Figure 5.

of $\vdash A$ (where $A$ is a formula without strong quantifiers) there exists a cut-free intuitionistic proof $\psi$ of $\vdash \neg \neg A$ s.t. $\|\psi\| \leq h(\|\varphi\|)$.

Proof: We extend $\varphi$ by a $\neg: l$ rule and obtain a cut-free proof $\varphi^{\prime}$ of $\neg A \vdash$. By Theorem 7 there exists a intuitionistic cut-free proof $\psi^{\prime}$ of $\neg A \vdash$ and $\left\|\psi^{\prime}\right\| \leq g(\|\varphi\|)$. We obtain an intuitionistic cut-free proof of $\neg \neg A$ just by appending $\neg: r$ to $\psi^{\prime}$. An elementary function $h$ with $\|\psi\| \leq h(\|\varphi\|)$ can be constructed from $g$ in an obvious way.

\section{CONCLUSION}

In this paper we describe an elementary transformation of cut-free LK proofs of skolemized sequents of the form $S: \Gamma \vdash$ into cut-free $\mathbf{L J}$ proofs of $S$. As a corollary, we get an elementary bound for Glivenko's double negation translation on sequents with weak quantifiers.

Concerning other double negation translations, we believe that, by using a suitable Skolemization of intuitionistic logic, the results of this paper may be extended to Kuroda translations. However, the Gödel-Gentzen-Kolmogoroff translation might not admit elementary bounds on translations of cutfree classical to cut-free intuitionistic proofs. A reason can be found, e.g., in the translation of Figure 5. We recall that cuts can be encoded as tautologies via $\rightarrow_{l}$ inferences, therefore any proof with cuts can be linearly translated to a cut-free proof of the same end-sequent extended by tautologies. By using the translation in Figure 5, the tautologies are removed, and this is equivalent to removing the cuts. Thus the transformation must be of non-elementary complexity. Of course, the calculi $\mathbf{L K}$ and $\mathbf{L J}$ should be extended by the axiom $\vdash T$. On the other hand, if the top clause of the case distinction is deleted the bound is linear.

It would be interesting to know more about the embeddings of logics on the proof theoretic level. The main result of this paper can be considered as a first step into the direction of comparing translations from analytic (in this case cut-free) to analytic proofs. The crucial tool for achieving the main result of this paper is the method CERES which, as a global method of proof analysis (compared to the local reductive ones), also allows a more global comparison of proofs.

\section{REFERENCES}

[1] K. Gödel, "Über die Länge von Beweisen," Ergebinisse eines mathematischen Kolloquiums (in German), vol. 7, pp. 23-24, 1936.

[2] S. R. Buss, "On Gödel's theorems on lenghts of proofs I: number of lines and speedup for arithmetics," J. Symb. Log., vol. 59, no. 3, pp. 737-756, 1994. [Online]. Available: http://dx.doi.org/10.2307/2275906

[3] — , "On Gödel's theorems on lengths of proofs II: lower bounds for recognizing k symbol provability," in Feasible Mathematics II, ser. Progress in Computer Science and Applied Logic, P. Clote and J. Remmel, Eds. Birkhäuser Boston, 1995, vol. 13, pp. 57-90.

[4] C. Smoryński, "The varieties of arboreal experience," The Mathematical Intelligencer, vol. 4, no. 4, pp. 182-189, 1982. [Online]. Available: http://dx.doi.org/10.1007/BF03023553

[5] A. Ehrenfeucht and J. Mycielski, "Abbreviating proofs by adding new axioms," Bull. Amer. Math. Soc., vol. 77, no. 3, pp. 366-367, 03 1971. [Online]. Available: http://projecteuclid.org/euclid.bams/1183532810

[6] D. Gabbay, Semantical Investigations in Heyting's Intuitionistic Logic, ser. Synthese Dordrecht / Library. Springer, 1981.

[7] M. Baaz and A. Leitsch, "Cut-elimination and redundancyelimination by resolution," Journal of Symbolic Computation, vol. 29 , no. 2 , pp. 149-176, 2000.

[8] G. Reis, "Cut-elimination by resolution in intuitionistic logic," Ph.D. dissertation, Vienna University of Technology, July 2014.

[9] M. Baaz and A. Leitsch, Methods of Cut-Elimination, ser. Trends in Logic. Springer, 2011.

[10] A. Leitsch, The resolution calculus, ser. Texts in theoretical computer science. Springer, 1997.

[11] M. Baaz and A. Leitsch, "Towards a clausal analysis of cutelimination," Journal of Symbolic Computation, vol. 41, no. 3-4, pp. 381-410, 2006. 